\title{
La valutazione degli esiti e del processo nelle psicoterapie offerte dai Centri di Salute Mentale e da un Centro di Psicologia Clinica universitario
}

\author{
Alessandra De Coro, ${ }^{1}$ Silvia Andreassi, ${ }^{1}$ Rachele Mariani, ${ }^{1}$ \\ Elisabetta Iberni, ${ }^{1}$ Valeria Crisafulli, ${ }^{1}$ Adriana Matarrese ${ }^{1}$
}

\section{Sommario}

Il presente lavoro si pone l'obiettivo di presentare i principali risultati emersi dalla valutazione empirica di esiti e di processo nelle psicoterapie condotte in contesti istituzionali. In una prima parte sono riassunti metodi e risultati di un progetto longitudinale articolato - che ha incluso un intervento di formazione e restituzione - svolto in collaborazione fra l'Università "Sapienza" e i Centri di Salute Mentale di una ASL romana. Successivamente sono illustrati gli studi condotti dal gruppo di ricerca sul cambiamento terapeutico e sul processo applicando strumenti di indagine basati sulle narrative alle sedute di psicoterapia presso un Centro Clinico Universitario. I principali obiettivi delle ricerche svolte sono rivolti al miglioramento dei servizi, all'auto-monitoraggio degli psicoterapeuti e all'elaborazione di un modello teorico che incentivi l'efficacia terapeutica.

\section{Parole chiave}

Psicoterapie istituzionali, valutazione esiti, ricerca sul processo, narrative, disturbi di personalità

\footnotetext{
${ }^{1}$ Dipartimento di Psicologia Dinamica e Clinica, "Sapienza" Università di Roma Corrispondenza: Alessandra De Coro

E-mail: Alessandra.decoro@uniroma1.i
} 


\section{Il lavoro del gruppo nei Centri di Salute Mentale}

Il gruppo di ricerca formato dalle Autrici, presso il Dipartimento di Psicologia dinamica e clinica dell’Università di Roma "La Sapienza", ha orientato dal 2002 il proprio lavoro di ricerca sull'efficacia delle psicoterapie nei servizi pubblici perseguendo tre obiettivi principali: in primo luogo, quello di introdurre dei protocolli di ricerca fondati su una metodologia adatta al funzionamento dei servizi e il più possibile condivisa dai professionisti (psicologi e psichiatri) che erogano in prima persona la psicoterapia; in secondo luogo, quello di individuare strumenti finalizzati alla diagnosi funzionale e di personalità nella prospettiva psicodinamica, che risultassero al tempo stesso user friendly per i pazienti e per i clinici e sufficientemente "oggettivanti" da permettere una valutazione attendibile del cambiamento terapeutico; in terzo luogo, l'obiettivo di mettere a punto strumenti di valutazione del processo che, accanto alla funzione di automonitoraggio da parte del terapeuta, promuovessero una riflessione più approfondita sulla natura stessa dei processi mentali attivati nel dialogo psicoterapeutico.

\section{La metodologia di ricerca per la valutazione delle psicoterapie nei contesti istituzionali}

La ricerca empirica sulla efficacia delle psicoterapie all'interno dei servizi pubblici riflette un cambiamento graduale nella cultura inerente la pratica clinica, avvenuto in concomitanza al progresso e allo sviluppo della metodologia scientifica in senso stretto. Nel 1999 le "Norme per la Razionalizzazione del Servizio Sanitario Nazionale", confermando l'impostazione di precedenti decreti, enfatizzavano l'importanza della funzione di verifica della qualità e di revisione dei risultati. Rispetto al settore delle psicoterapie, l'applicazione di queste normative ha sollecitato una maggiore sinergia tra diversi attori istituzionali, come, da un lato, il Ministero della Sanità, le Regioni e i Dipartimenti di Salute Mentale e, dall'altro lato, gli organi tecnico-scientifici, afferenti 
rispettivamente alle ASL e alle Università o ad altri enti di ricerca. Le attività di ricerca del gruppo, in riferimento al primo obiettivo indicato, hanno cercato di supportare il cambiamento organizzativo e culturale di alcune ASL di Roma (in particolare la Rm B, coinvolta con due CSM, e poi, più estesamente, la $\mathrm{Rm} \mathrm{A}$, con cui è tuttora in corso una convenzione di ricerca), attraverso l'impostazione di progetti di valutazione dell'efficacia dei trattamenti: tali progetti hanno sempre presentato caratteristiche di ricerca di tipo "ecologico" o "naturalistico", non raggiungendo gli standard richiesti dal paradigma epistemologico dell'Evidence Based Mental Health (De Coro \& Andreassi, 2004). La ricerca sull'efficacia nei contesti istituzionali presenta infatti problemi insormontabili rispetto all'esigenza di standardizzare le situazioni osservate, come per esempio: l'eterogeneità dell'utenza; la natura variabile e non prefissata della durata dei trattamenti; la comorbidità e la frequenza di doppie diagnosi; la molteplicità dei percorsi formativi dei clinici e le diverse tecniche d'intervento, spesso cucite "su misura" rispetto alle specificità dei pazienti e del setting istituzionale. Pur nella consapevolezza dei limiti di questa metodologia e della reale generalizzabilità dei risultati (Dazzi, 2006), la nostra pianificazione degli interventi di valutazione delle psicoterapie ha cercato di bilanciare in maniera ottimale le esigenze di minore intrusività della ricerca nel lavoro clinico con l'esigenza di utilizzare misure quantitative e ripetute nel tempo.

Un primo progetto di ricerca biennale in collaborazione con l'ASL Roma B è stato incentrato sull'uso di questionari self-report, somministrati sia ai pazienti che ai terapeuti, con ritmo semestrale. Al terapeuta era richiesta la diagnosi clinica secondo l'ICD-9 e la compilazione della PRS (Scala di Valutazione Periodica dell'intervento psicoterapeutico, adattata in italiano da Gherardo Amadei); al paziente la compilazione di un questionario sulla frequenza e intensità dei sintomi presenti nelle ultime due settimane (SCL-90) e, dopo un anno, la valutazione della propria 
soddisfazione per l'intervento ricevuto (ROS-SC: Scala Romana Autocompilata sulla Opinione del paziente); entrambi, infine, dovevano monitorare la relazione reciproca compilando la WAI, una scala per l'alleanza terapeutica (De Bei, 2006). L'esperienza di questo primo progetto ha evidenziato alcuni limiti della metodologia scelta e le difficoltà della valutazione delle psicoterapie nei servizi, malgrado la volonterosa collaborazione dei colleghi terapeuti e l'impegno messo dal nostro gruppo nella stesura di report semestrali indirizzati ai clinici per il monitoraggio dei singoli casi ${ }^{1}$. La presenza di frequenti drop-out, infatti, ha ridotto la numerosità del gruppo di psicoterapie studiato fino a un anno (25 su 40 soggetti inclusi nello studio); l'applicazione della PRS a tanta distanza di tempo risultava poco utilizzabile per il monitoraggio del processo. Dati interessanti erano invece quelli sull'alleanza terapeutica, che indicava una buona capacità predittiva rispetto all'esito della psicoterapia, in termini di modificazione del quadro sintomatologico e di soddisfazione del paziente rispetto al servizio: in particolare, la qualità della relazione riferita dal terapeuta nella WAI risultava significativamente correlata con aggravamenti e miglioramenti segnalati dal paziente sulla SCL-90 (Matarrese, 2007).

Contemporaneamente, per migliorare la sensibilità della diagnosi iniziale, il gruppo ha lavorato all'adattamento italiano dell'OPD (Diagnosi Psicodinamica Operazionalizzata: Gruppo OPD, 1996-2001; Task Force OPD, 2006), uno strumento per la valutazione in termini strutturali e relazionali dei colloqui clinici, pubblicato nel 2001 in Germania da un gruppo di clinici e di ricercatori tedeschi: Gruppo OPD, 2001). ${ }^{2}$ In particolare, uno studio sulla convergenza fra OPD e diagnosi clinica, condotto in collaborazione con un centro privato di Milano, ha

\footnotetext{
${ }^{1}$ Si ringraziano il primario psichiatra, prof. Antonello Correale, e i dottori Vincenzo Scala e Giuseppe Di Leone, per aver promosso il progetto e mantenuto attiva la collaborazione dei colleghi psicoterapeuti.

2 Nel 2006 è uscita una seconda versione, modificata per migliorarne le caratteristiche psicometriche e la validità clinica. Dal 2009, questa edizione è disponibile in lingua italiana, a cura di Martina Conte, Emilio Fava e coll., per i tipi della Franco Angeli.
} 
evidenziato che l'asse struttura dell'OPD riesce a cogliere l'organizzazione della struttura psichica e che si riscontrano convergenze con alcune valutazioni del test di Rorschach (De Coro, Lang, Del Corno, Parolin, Matarrese, Piscicelli, Iberni, \& Basile, 2004). L'OPD ha mostrato anche buone potenzialità per essere utilizzato nell'ambito di un assessment finalizzato alla diagnosi funzionale ed alla pianificazione di trattamenti ad hoc per diversi gruppi di pazienti (Iberni, Vicari, Tagini, \& Pazzagli, 2004).

Nel 2007 è stato firmato un accordo di cooperazione con la ASL Rm A per un progetto di ricerca sulla valutazione del processo e dell'esito delle psicoterapie, esteso a cinque Centri di Salute Mentale, creando una sinergia con il Dipartimento universitario.

Sulla base delle lessons learnt della precedente esperienza, il protocollo di questa valutazione ha incrementato l'uso di strumenti più articolati e ricchi di informazioni sul piano dell'osservazione clinica, come l'Intervista sull'attaccamento adulto rivolta alla/al paziente (cfr. Speranza, 2006) e la SWAP-200 (cfr. Gazzillo, 2006) somministrata alla/al terapeuta dopo la fase dei colloqui iniziali; entrambe le somministrazioni sono ripetute dopo un anno. Il gruppo di pazienti inclusi nella ricerca sono coloro che dopo essere stati valutati con una diagnosi psichiatrica e con il livello di funzionamento globale (VGF), decidono di iniziare una psicoterapia (individuale o di gruppo), sia in combinazione con un trattamento farmacologico che senza; sono esclusi i soggetti che hanno avuto diagnosi di tossicodipendenza e che presentano disturbi di tipo organico (Iberni, Crisafulli, \& De Coro, 2007). Ai terapeuti è stata richiesta anche la compilazione della PRS una volta al mese, per avere maggiori possibilità di riscontrare variazioni di processo, mentre i pazienti compilano tre questionari carta e matita: la Symptom Check List-90-Revised (SCL-90-R: Derogatis, 1977); la Response Evaluation Misure-71 (REM-71; Steiner, Araujo, Koopman, 2001; Lingiardi, 2006); il Coping Orientation to Problems Experienced 
(COPE; Carver, C. S., Scheier, M. F., \& Weintraub, J. K., 1989) che valuta gli stili di adattamento e le strategie per fare fronte allo stress. Le rilevazioni di questi dati vengono ripetute a sei mesi, mentre l'AAI e la SWAP-200 vengono somministrate nuovamente solo dopo un anno. I trattamenti brevi (entro i 6 mesi) e i casi di drop out vengono ricontattati per un follow-up dopo un anno dall'inizio della terapia per la somministrazione degli strumenti della Fase 4 (dopo un anno).

Attualmente la ricerca include 41 pazienti che afferiscono a tre Centri di salute mentale della ASL Roma A e 7 terapeuti diversi, prevalentemente con orientamento psicodinamico. ${ }^{3}$ La diagnosi clinica risulta, nel 35,3 \% dei casi, quella di Disturbo dell'Umore e nel 13,7\% di Disturbo di Adattamento o Postraumatico da Stress. Sul totale, in 14 casi si è riscontrato un drop out prima dei sei mesi di terapia; 15 psicoterapie hanno superato i sei mesi di terapia e 9 hanno superato un anno: solamente 3 pazienti, finora, hanno concluso in modo concordato la terapia. I primi risultati emersi dalle due somministrazioni dei self report (SCL-90, REM-71 e COPE), in prima valutazione e dopo sei mesi di trattamento, non mostrano nessun cambiamento significativo.

Rispetto alla valutazione dello stato della mente relativo all'attaccamento, alla prima AAI il 46\% dei pazienti mostra una disorganizzazione, mentre fra gli altri prevalgono i soggetti Sicuri $(\mathrm{F}=25 \%)$, poi gli Invischiati $(18 \%)$ e infine i Distanzianti, che sono in percentuale inferiore alla distribuzione nelle popolazioni normali $(7 \%$ contro i dati normativi che si aggirano intorno al 18-20\%). Il gruppo dei soggetti "Drop Out" si distingue per un maggior ricorso al meccanismo di difesa della Fantasia, per un uso maggiore di Meccanismi Evasivi e per tratti Narcisistici significativamente più marcati rispetto agli altri soggetti. I pazienti con uno stato della mente Sicuro usano più intensamente meccanismi di difesa Evasivi rispetto agli Insicuri, che

3 Si ringraziano il primario psichiatra, Prof. Giorgio Campoli, che ha avviato e sostenuto l'iniziativa e gli psicologi clinici dott. Ennio Fusco, dott. Giuseppe Mancini e dott.ssa Leonella Magagnini, che, in quanto referenti dei rispettivi Distretti, hanno collaborato attivamente alla ricerca in tutte le sue diverse fasi. 
mostrano dei tratti di personalità Antisociale significativamente più marcati dei Sicuri. Anche le strategie di coping sembrano distribuirsi diversamente in relazione ai diversi stati della mente relativi all'attaccamento.

Per quanto riguarda la diagnosi di disturbo di personalità effettuata con la SWAP-200, sembra che i soggetti con una diagnosi accertata facciano maggiormente uso di sostanze rispetto a coloro che non hanno ricevuto una diagnosi di personalità. I pazienti con tratti schizoidi e schizotipici ricorrono più frequentemente fra coloro che sono classificati non risolti/disorganizzati all'AAI.

Secondo questi dati descrittivi, le variabili Attaccamento e Disturbo di Personalità risultano associate alla continuità della terapia e sembrano offrire una particolare utilità nella pianificazione del trattamento per la valutazione dei modelli relazionali del paziente. Analogamente alla prima esperienza, la verifica dell'esito e del cambiamento dei pazienti dipende essenzialmente dalla durata del trattamento oltre i 6 mesi. Rispetto al cambiamento terapeutico, la ricerca ha permesso di valutare qualitativamente 9 casi, che hanno migliorato non solo i sintomi manifesti, ma anche le strategie di coping, il grado di funzionamento globale e la disorganizzazione dell'attaccamento. L'esiguità del numero, tuttavia, non ha ancora consentito di trattare statisticamente i risultati in modo soddisfacente.

Nello stesso progetto è stato introdotto recentemente anche uno studio rivolto alla valutazione dei pazienti gravi, utenti delle strutture che accolgono pazienti con diagnosi di schizofrenia e altre forme psicotiche. L'obiettivo ultimo è quello di monitorare l'efficacia degli interventi di riabilitazione psico-sociale, ma la prima fase è stata dedicata alla valutazione psicodinamica multi- dimensionale, includendo l'utilizzo dell'OPD (in forma di questionario rivolto agli operatori che lavorano nel Centro diurno), e la somministrazione di tre questionari al paziente, rivolti a rilevare la sintomatologia (SCL-90-R), i tratti del carattere (BFQ: 
Big Five Questionnaire, adattato da Caprara, Barbaranelli, Borgogni, \& Perugini, 1993), il grado di compromissione dell'organizzazione strutturale della personalità (IPO: Inventory of Personality Organization, Kernberg \& Clarkin, 1995, non ancora validato in Italia) e infine le risorse del paziente, misurate attraverso un questionario sul benessere percepito (PWB: Psychological Well-Being Scales, adattato da Ruini, Ottolini, Rafanelli, Tossani, Ryff, \& Fava (2003). Fino a questo momento 15 utenti hanno partecipato alla ricerca, pazienti di un Centro Diurno della Roma A che frequentano in media da 5 anni il servizio che hanno ricevuto una diagnosi di schizofrenia o altra forma psicotica. Il lavoro sta proseguendo in una Comunità Terapeutica.

\section{La diagnosi strutturale e la validazione dell'Inventory of Personality Organization}

L'Inventory of Personality Organization (IPO; Kernberg \& Clarkin, 1995)

è un self-report che permette di misurare l'organizzazione strutturale della personalità del soggetto e di iscriverla, sulla base della sua compromissione, ad un livello nevrotico, borderline o psicotico. E' uno strumento che permette di implementare il colloquio clinico-strutturale di Kernberg (1984) e di effettuare uno screening iniziale sulla popolazione clinica, che possa aiutare a distinguere ed identificare i casi più compromessi da quelli che lo sono di meno, in modo poco oneroso. Obiettivo è quello di dare un'adeguata indicazione di trattamento nei contesti in cui la domanda è copiosa e le risorse sono limitate. Nella sua forma originale l'IPO conta 155 item divisi nei cinque criteri strutturali: Diffusione d'Identità, Difese Primitive, Esame di Realtà; Aggressività e qualità delle Relazioni Interpersonali; la forma abbreviata (l'unica attualmente pubblicata e validata da Lenzeweger, Clarkin, Kernberg, \& Foelschet, 2001) considera solo le prime tre dimensioni riportate, attraverso 57 item. Le tre scale misurano quanto un paziente presenti un' identità diffusa, quanto utilizzi difese primitive e quanto sia 
compromesso il suo esame di realtà. Dall'analisi delle tre dimensioni si possono identificare le tre categorie strutturali nevrotica, borderline e psicotica.

Il lavoro svolto mira all'adattamento italiano dell'IPO, attraverso la valutazione delle caratteristiche psicometriche dello strumento tradotto e applicato alla popolazione italiana (Crisafulli, in preparazione). Dai primi risultati su un campione non clinico di 470 soggetti è risultata una nuova struttura fattoriale: le scale sono sempre tre, ma gli item si suddividono in modo differente dalla struttura originaria. Dal contenuto degli item abbiamo definito i tre nuovi fattori: Distorsione dell'immagine di sé, Distorsione dell'immagine dell'altro e Distorsione della realtà. La nuova struttura è stata analizzata, parallelamente a quella originaria per determinarne le caratteristiche di validità e attendibilità. Dai primi dati emerge che entrambe mostrano buone qualità psicometriche. Per approfondire lo studio dello strumento stiamo raccogliendo anche un campione della popolazione clinica italiana: suddivisa in soggetti con disturbi o sindromi psicologiche non psicotiche, soggetti con disturbi di alcolismo o tossicodipendenza e soggetti con diagnosi di schizofrenia o altre forme psicotiche. Abbiamo un gruppo di 64 soggetti che stiamo implementando. Dalle analisi su questo primo campione ci risulta che la struttura originaria dell'IPO, meglio di quella nuova emersa dall'analisi dei risultati del gruppo italiano, discrimina i soggetti clinici da quelli non clinici e i soggetti psicotici da quelli non psicotici; di questi ultimi il fattore più discriminante è proprio l'esame di realtà.

Nonostante lo studio sia ancora incompleto, i primi risultati confermano le buone capacità psicometriche dell'IPO, rilevate già dai lavori americani, anche rispetto a soggetti italiani. I dati mostrano e confermano le basi del costrutto teorico di riferimento, il modello della struttura di personalità di Kernberg, evidenziando come sia importante accanto ad una valutazione categoriale o dimensionale anche quella strutturale affinché i dati empirici possano avere anche rilievo clinico. 


\section{Strumenti per una valutazione delle narrative}

Nell'ambito di uno studio sul processo intrapreso sulle psicoterapie individuali ad orientamento psicodinamico condotte presso il Centro clinico del Dipartimento universitario, con l'ausilio di audio-registrazioni delle sedute, sono stati adattati alla lingua italiana nuovi strumenti di valutazione, rilevanti per il monitoraggio dei micro-cambiamenti nel corso del processo e utili ad una riflessione sulla natura degli scambi verbali e non verbali che si verificano in psicoterapia.

\section{La coerenza narrativa come parametro del cambiamento dei modelli operativi interni di attaccamento}

La ricerca sulla coerenza narrativa come parametro utile alla valutazione e misurazione della sicurezza dello stato della mente rispetto all'attaccamento adulto è stata sviluppata sin dall'inizio degli anni '90 (Muscetta, Dazzi, De Coro, Ortu, \& Speranza, 1999). L'esigenza di sviluppare una nuova misura dimensionale per la valutazione dell'attaccamento adulto è stata sollevata recentemente da alcuni autori che hanno evidenziato le lacune metodologiche ed il loro impatto sulla concettualizzazione teorica dell'attaccamento del sistema categoriale (Roisman, Fraley, \& Belsky, 2007). Il Coherence Q-Sort (Beijersbergen, Bakermans-Kranenburg, \& Van Ijzendoorn, 2006) è uno strumento composto da 72 items, che operazionalizza la scala della coerenza del trascritto considerata fondamentale per l'attribuzione dello stato della mente relativo all'attaccamento negli adulti. La validazione italiana di questo strumento ha mostrato una sostanziale capacità di discriminare $i$ trascritti sicuri da quelli insicuri (Iberni, 2010). Da circa trent'anni l'Adult Attachment Interview (AAI) (George, Kaplan, \& Main, 1985) è lo strumento preferenziale per la valutazione dell'attaccamento adulto, che utilizzate il complesso sistema di codifica tradizionale poi aggiornato (Main \& Goldwyn, 1998; Main, Hesse \& Goldwyn, 2008). Inizialmente il 
sistema di scoring e di classificazione era basato sulla distinzione fra le tre categorie di attaccamento infantile misurate nella Strange Situation, ponendo enfasi sul concetto di flessibilità attentiva, intesa come capacità di presentare le proprie esperienze di attaccamento passate e presenti e di valutarne la loro influenza sul proprio sviluppo in maniera fluida e spontanea. La sicurezza dell'attaccamento, tradotta in termini di flessibilità attentiva, vede all'opposto due forme di rigidità nel focalizzare l'attenzione: la prima distanziante, in cui il discorso si allontana dalle esperienze passate e dalle relazioni di attaccamento; la seconda preoccupata/ invischiante, in cui il focus sulle esperienze di attaccamento è persistente ma confuso, ridondante e di difficile comprensione. Questi pattern di risposta possono essere tradotti in pattern di organizzazione del discorso a partire dalla teoria del linguista Grice (1975), che ha introdotto il concetto di Principio di Cooperazione dei parlanti secondo l'accordo implicito di rispettare quattro massime o principi generali: Qualità, Quantità, Rilevanza e Stile.

Il Coherence Q-sort è uno strumento applicabile nella valutazione del cambiamento dei modelli operativi interni durante e dopo la psicoterapia. I risultati di precedenti ricerche avevano già illustrato le potenzialità della coerenza narrativa per la valutazione del cambiamento durante e dopo il trattamento psicoterapeutico: in particolare alcuni studi italiani che hanno applicato in ambito clinico le massime griciane della coerenza (Muscetta et al., 1999). I risultati empirici della ricerca condotta sulle AAI dei pazienti della ASL Roma A hanno messo in luce come, più che il cambiamento della categoria dell'attaccamento, sia interessante il cambiamento dimensionale della coerenza narrativa (Iberni, 2010). Il fatto che la coerenza distingua gruppi clinici e non clinici e costituisca il parametro più predittivo del cambiamento degli stati mentali relativi all'attaccamento durante la psicoterapia è stato confermato da diversi studi (Diamond, Clarkin Levine, Levy, Foelsch, \& Yeomans, 1999; Fonagy, Gergely, Jurist \& Target 2002). 


\section{Attività Referenziale e dizionari IWRAD}

Un altro studio sul processo terapeutico è stato condotto da diversi anni con l'applicazione della valutazione dell'Attività Referenziale, costrutto derivato dalla Teoria del Codice Multiplo di Wilma Bucci (1997), che integra ricerca empirica e lavoro clinico. La ricerca in psicoterapia basata sulla valutazione dell'Attività Referenziale in Italia nasce alla fine degli anni ' 90 , grazie alla traduzione del metodo manuale di siglatura della variabile che ne ha permesso l'applicazione e la diffusione (De Coro \& Caviglia, 2000). Alcuni anni dopo, gli indirizzi di ricerca proposti dall'ideatrice del metodo hanno direzionato la ricerca verso la costruzione di un metodo di più facile applicazione, rendendo la valutazione della variabile Attività Referenziale una codifica informatizzata. In Italia, quindi, il gruppo di ricerca coordinato dalla Prof.ssa De Coro, dal 2004 ha collaborato con l'Adelphi University allo sviluppo delle misure computerizzate in lingua italiana. Attualmente sono state sviluppate in italiano le medesime misure prodotte da Bucci e Maskit in lingua inglese. Il primo dizionario prodotto è stato quello dell'IWRAD, Italian Weighted Referential Activity Dictionary (Maskit, 2004; De Coro, Ortu, Caviglia, Andreassi, Pazzagli, Mariani, Visconti, Bonfanti, Bucci \& Maskit, 2004) unico nella sua metodologia di costruzione, che consente di attribuire ad ogni parola appartenente al dizionario uno specifico peso ponderato. Questa procedura ha permesso di costruire un dizionario con una vasta copertura del linguaggio parlato, in media del $98 \%$ delle parole espresse in un qualsiasi scambio comunicativo. Più recenti sono i dizionari di analisi del testo che possono essere applicati contemporaneamente all'IWRAD e che riguardano: il dizionario delle parole riflessive (IREF- Mariani, 2009); il dizionario della difluenza (IDF - Bonfanti, Campanelli, Cilimberti, Golia, \& Papini, 2008); il dizionario degli affetti positivi, negativi e parole con peso affettivo ma non connotate positivamente o negativamente (IAFF - Rivolta, Mariani, \& 
Tagini 2009); infine il dizionario senso-somatico (ISS - Mariani, 2009). La facilità di applicazione delle misure computerizzate ai testi trascritti ha consentito una rapida applicazione di queste misure in diversi ambiti, consentendo uno sviluppo della ricerca sul processo referenziale in relazione ad altri costrutti teorici, nonché il suo ambito storico ossia la ricerca sul processo in psicoterapia. Anche rispetto a quest'ultimo è stato possibile poter analizzare con più semplicità interi percorsi di psicoterapia di più anni, consentendo una valutazione del processo referenziale in un ottica di macro-processo terapeutico. I risultati emersi (Mariani, 2009) dall'applicazione di tre dizionari computerizzati - IWRAD, IREF e IDF - su tre macro processi terapeutici della durata di due anni, rilevano la presenza di macro cicli referenziali trasversalmente alle sedute, comportando un aumento di capacità del paziente di connettere emozioni al tessuto narrativo. Un ulteriore risultato emerge dalla possibilità di analizzare le sedute grazie all'applicazione del dizionario IWRAD (Attività Referenziale) e IREF (Parole Riflessive). La combinazione di queste due misure consente di individuare un indice di covariazione utile a distinguere sedute proficue, nelle quali il paziente è in grado di raccontare e riflettere sulla propria esperienza, e sedute più disorganizzate nelle quali l'attivazione emozionale blocca il processo di simbolizzazione.

L'utilizzo delle misure linguistiche per l'analisi del macroprocesso terapeutico ha consentito lo sviluppo di un altro filone di ricerca che consente di incrociare misure sul processo e misure sull'outcome. Lo studio di Mariani, Gazzillo, Lingiardi e Bucci (2009) propone un'analisi pilota che integra misure di valutazione della personalità SWAP (Westen \& Shedler, 2000; Lingiardi, Shedler, \& Gazzillo, 2006) e misure linguistiche del processo referenziale in un caso singolo. Lo studio si basa sull'analisi di una psicoterapia a orientamento psicodinamico, dove sono state applicate tre misure linguistiche computerizzate: Attività Referenziale (IWRAD), Parole Riflessive (IREF) e Disfluenza (IDF). Sono 
state individuate delle fasi di cambiamento e per ogni fase la paziente è stata valutata con la SWAP. Sono state estratte 4 sedute consecutive per fase e ogni fase è stata siglata in modo random da due valutatori della SWAP. I risultati mostrano che, per ognuna di queste fasi, è possibile individuare cambiamenti significativi nel profilo di personalità valutato con la SWAP.

Un'altra area di indagine, sempre all'interno dello studio sul processo terapeutico, è l'esplorazione della relazione tra narrazione e alleanza terapeutica. Nello studio di Colli, Mariani, Condino, Lingiardi e De Coro (2009) si esplora il rapporto tra il processo referenziale (Bucci, 1997) e i processi di rottura e di riparazione dell'alleanza terapeutica secondo il modello teorico clinico descritto da Safran e Muran (2000). In particolare, è stata indagata la relazione tra le misure linguistiche del processo referenziale, quali la scala dell'Attività Referenziale (IWRAD), delle parole Riflessive (IREF), della Disfluenza (IDF),e degli Affetti (IAFFP e IAFFN), e i processi di rottura dell'alleanza di Ritiro e di Confrontazione. Le analisi sono state compiute su di un campione di 12 sedute audioregistrate, scelte all'interno di un campione più ampio, in quanto caratterizzate dalla presenza di riferimenti espliciti alla relazione terapeutica. La valutazione dei processi di rottura e riparazione dell'alleanza terapeutica è stata effettuata attraverso la Collaborative Interaction Scale (Colli \& Lingiardi, 2009). I risultati evidenziano come i processi collaborativi sono risultati correlati significativamente e positivamente alla misura delle parole Riflessive e dell'espressione degli Affetti negativi. Mentre marker di rottura di Confrontazione sono risultati correlati in modo significativo e diretto ai valori della Disfluenza e all'espressione di Affetti negativi e negativamente alla scala delle parole Riflessive. Le rotture di ritiro sono risultate correlate negativamente all'espressione di Affetti di tipo negativo e positivamente alla Disfluenza. Ciò indica una convergenza tra i due costrutti e l'importanza di mettere in relazione i processi di rottura in relazione ai processi di negoziazione 
intersoggettiva tra paziente e terapeuta, sia in relazione ai processi di disconnessione emozionale emersa dalla narrazione.

La possibilità di incrociare i risultati ottenuti con le misure linguistiche con altre misure di indagine sul processo ha portato recentemente ad uno studio single case, una psicoterapia breve, sul quale vengono applicate diversi strumenti di analisi delle narrazioni. I risultati, presentati al convegno 2010 dell'SPR Italia nel panel proposto da Rocco, Mariani, De Bei, Colli, e De Coro "Analisi multidimensionale del processo in una psicoterapia dinamica breve", hanno evidenziato come sia possibile utilizzare misure sul processo terapeutico anche a psicoterapie brevi, riuscendo a mettere in evidenza diversi aspetti del cambiamento, grazie alla specificità dei diversi strumenti nella lettura dei trascritti.

Infine parallelamente allo studio del processo in psicoterapia, lo studio del processo referenziale e della capacità soggettiva di integrare nel tessuto simbolico i propri affetti sta espandendosi verso studi correlazionali che esplorano come la variabile Attività Referenziale possa essere anche utilizzata come una variabile di tratto soggettivo e come questa possa essere posta in relazione ad altre misure di funzionamento strutturale del soggetto. In particolare, sono state applicate per la prima volta misure linguistiche del processo referenziale alle narrazioni di bambini in età dello sviluppo (Mariani, Pazzagli, Crisafulli, \& De Coro, 2010) cercando di esplorare la relazione tra variazioni delle capacità linguistiche nei bambini di età compresa tra gli 8 e i 12 anni e il rapporto tra narrazione e attivazione del sistema motivazionale di attaccamento. L'obiettivo è quello di approfondire la valutazione delle narrative in relazione alle capacità del bambino di esprimere le emozioni e di verificare se la relazione di attaccamento faciliti, o viceversa ostacoli nei casi di insicurezza, lo sviluppo dell'abilità di elicitare affetti e di attivare una capacità referenziale. Lo scopo del lavoro, quindi, è verificare se i bambini con attaccamento sicuro presentino una maggiore abilità nell'integrare gli affetti nel processo narrativo rispetto ai bambini 
con attaccamento insicuro, all'interno di un campione non clinico. Sono state per tanto raccolte e trascritte 100 narrazioni eliticitate dalle tavole proiettive del SAT (Klagsbrun \& Bowlby, 1976). Sui trascritti delle narrative sono state applicate diverse misure linguistiche dell'Attività Referenziale come: Attività Referenziale (IWRAD), Dizionari Affetti (IAFF), Parole Riflessive (IREF), Disfluenza (IDF). I risultati mostrano una differenza significativa tra bambini con attaccamento sicuro ed insicuro nella capacità di esprimere affetti e nell'abilità di narrare l'esperienza integrando le emozioni nel tessuto narrativo.

\section{Riflessioni conclusive}

I filoni di ricerca qui sommariamente illustrati fanno riferimento ad un comune denominatore, la ricerca empirica in psicoterapia, che si può a sua volta articolare in alcune linee guida che hanno ispirato il nostro lavoro:

a) l'esigenza di costruire progetti per la verifica dell'outcome che rispettino la natura variegata dell'esperienza clinica e della pratica "reale" della psicoterapia nei servizi pubblici: a questo scopo sono stati inclusi nella valutazione pazienti diversi e diversi tipi di psicoterapie, ed una gran parte del lavoro è stata diretta alla restituzione delle valutazioni sia ai pazienti che ai terapeuti con modalità di volta in volta concordate con i clinici, rispettando tradizioni e sensibilità specifiche dei diversi servizi;

b) l'uso di batterie multi-strumentali per raggiungere una maggiore validità della misurazione grazie alla convergenza degli indicatori: in particolare, l'introduzione di valutazioni da parte dei clinici accanto alle auto-valutazioni dei pazienti si è rivelata la scelta migliore per una maggiore validità clinica dei dati e per una riflessione sulla natura relazionale del lavoro psicoterapeutico;

c) la messa a punto di strumenti per la valutazione iniziale del paziente che riflettano la complessità delle situazioni cliniche e permettano la 
replicabilità della misurazione in fasi successive del trattamento e alla fine della psicoterapia; la validazione di strumenti self-report, più veloci nella somministrazione e certamente più attendibili nella misura, è stata affiancata dall'adattamento italiano di strumenti molto complessi per lo studio del processo, basati sulla micro-analisi delle narrative, come il dizionario computerizzato dell'attività referenziale e il Q-sort per la valutazione della coerenza narrativa: tali strumenti, meno attendibili dei questionari, risultano però incrementare la validità di costrutto e quindi l'interpretazione del significato clinico dei risultati ottenuti.

Fra i limiti delle ricerche riportate, segnaliamo in particolare la lentezza del processo di raccolta dei dati nei servizi pubblici e le difficoltà di compliance da parte dei terapeuti nel compilare i questionari loro richiesti, per la mole di lavoro routinario che spesso distoglie i clinici dei centri di salute mentale dal dedicare del tempo alla ricerca ed alla riflessione sul proprio lavoro. D'altra parte, l'entusiasmo con cui tutti i terapeuti (o quasi) dei diversi servizi coinvolti hanno accolto l'iniziativa induce a riporre speranze nella possibilità di estendere la cultura della ricerca nei Centri di salute mentale, affinando metodi e procedure così da ridurne l'impatto per il tempo degli operatori clinici: la sinergia con l'università sembra dare promettenti auspici in questa direzione, perché una parte del lavoro di raccolta dei dati può essere svolto da tirocinanti, laureandi o dottorandi con un raccordo centralizzato, pur nel rispetto delle specifiche esigenze di funzionamento dei diversi servizi.

Si può concludere ricordando che, mentre gli obiettivi che si poneva la ricerca in psicoterapia nella sua fase iniziale erano identificati soprattutto con la validazione di specifiche tecniche per la cura di specifici disturbi, i risultati ottenuti grazie ai progressi della ricerca in questo settore hanno contribuito ad orientare i clinici verso la scelta di modelli integrati di intervento e a spingere $\mathrm{i}$ ricercatori a focalizzare l'attenzione sui fattori comuni del cambiamento terapeutico. Le ipotesi 
relative ai fattori responsabili del cambiamento, pur nella difficoltà di rispondere in modo empirico ad una domanda così complessa, vertono oggi sempre più sulle qualità della relazione terapeutica: più precisamente, la qualità e l'efficacia degli interventi, in qualsiasi forma tecnica siano espressi, è da valutarsi in relazione alla capacità del terapeuta di cogliere il focus tematico e di approfondirne la discussione (Colli \& Gazzillo, 2006). A questo scopo, la valutazione iniziale necessita di un'articolazione complessa, utilizzando strumenti che permettano di accostare, alla valutazione oggettiva e comparata della psicopatologia, delle ipotesi circa l'organizzazione strutturale della personalità del paziente e le sue specifiche vulnerabilità rispetto alle relazioni interpersonali. A nostro avviso, l'introduzione del registratore nelle psicoterapie offerte dai servizi pubblici - ovviamente nel rispetto della privacy di paziente e terapeuta - potrebbero implementare al meglio la valutazione del cambiamento terapeutico con la valutazione del processo attraverso il quale il cambiamento è stato raggiunto.

\section{Bibliografia}

Beijersbergen, M.D., Bakermans-Kranenburg, M.J., \& Van IJzendoorn, M.H. (2006). The concept of coherence in attachment interviews: Comparing attachment experts, linguists, and non-experts. Attachment \& Human Development, 8, 353-369.

Bonfanti A.A., Campanelli L., Ciliberti A., Golia G., \& Papini S.P. (2008, Giugno) Speech disfluencies in spoken language: The Italian Computerized Dictionary (I-Df) and its application on a single case. Paper presented at the SPR International Meeting, Barcellona.

Bucci, W. (1997) Psicoanalisi e Scienza Cognitiva, Roma: G. Fioriti, 1999.

Caprara, G.V., Barbaranelli, C., Borgogni, L., \& Perugini, M. (1993). The "Big Five Questionnaire": a new questionnaire to assess the Five Factor Model. Personality and Individual Differences, 15, 281-288.

Carver, C.S., Scheier, M.F., \& Weintraub, J.K. (1989). Assessing coping strategies: A theoretically based approach. Journal of Personality and Social Psychology, 56, 267-283. 
Colli, A., \& Gazzillo, F. (2006). Due strumenti per la valutazione del processo terapeutico: le Analytic Process Scales e lo Psychotherapy Process Q-Set. In N. Dazzi, V. Lingiardi \& A. Colli (Eds.), La ricerca in psicoterapia. Modelli e strumenti (pp. 677-689). Milano: Raffaello Cortina.

Colli, A., \& Lingiardi, V. (2009). The Collaborative Interactions Scale: A new transcript-based method for the assessment of therapeutic alliance ruptures and resolutions in psychotherapy. Psychotherapy Research, 19, 718-734.

Condino, V., Colli, A., Mariani, R., \& De Coro, R. (2009, Settembre). Studio sui processi di rottura dell'alleanza terapeutica in relazione alle comunicazioni emozionali nel processo referenziale. Relazione presentata al Convegno Nazionale AIP - Sezione Clinica, Chieti.

Crisafulli, V. (in preparazione), Verso la standardizzazione italiana dell' Inventory of Personality Organization: uno studio di fase 1. Tesi di Dottorato in Psicologia Dinamica, Clinica e dello Sviluppo. "Sapienza" Università di Roma.

Dazzi, N. (2006). Il dibattito contemporaneo sulla ricerca in psicoterapia. In N. Dazzi, V. Lingiardi \& A. Colli (Eds.), La ricerca in psicoterapia. Modelli e strumenti (pp. 3-29). Milano: Raffaello Cortina.

De Bei F. (2006). La ricerca sull'alleanza terapeutica. In N. Dazzi, V. Lingiardi \& A. Colli (Eds.), La ricerca in psicoterapia. Modelli e strumenti (pp. 505537). Milano: Raffaello Cortina.

De Coro, A., \& Andreassi, S. (2004). La ricerca empirica in psicoterapia. Roma: Carocci.

De Coro, A., \& Caviglia, G. (Eds.). (2000). La valutazione dell'Attività Referenziale. Roma: Kappa.

De Coro, A., Lang, M., Del Corno, F., Parolin, L., Matarrese, A., Piscicelli, S., Iberni, E., \& Basile, M. (2004, Giugno). Operationalized psychodynamic diagnosis: A study of convergence between OPD system and clinical diagnosis. Paper presented at the 35th Annual Meeting SPR, Rome, Italy. Book of Abstracts, Ulmer Textbank, Ulm, p. 69.

De Coro, A., Ortu, F., Caviglia, G., Andreassi, S., Pazzagli, C., Mariani, R., Visconti, E., Bonfanti, A., Bucci, W., \& Maskit, B. (2004, Giugno). Referential Activity and Verbal Exchanges in Psychotherapy: A First Clinical Application of the Italian WRAD Version to the Study of a Single Case. Paper presented at $35^{\text {th }}$ Annual Meeting of the Society for Psychotherapy Research, Rome.

Derogatis, L.R. (1977). The SCL-90-R: Administration scoring and procedures, Manual I. Baltimore: Clinical Psychometric Research.

Diamond, D., Clarkin, J., Levine, H., Levy, K., Foelsch, P., \& Yeomans, F. (1999). Borderline conditions and attachment: A preliminary report. Psychoanalytic Inquiry, 19, 831-884.

Fonagy, P., Gergely, G., Jurist, E., \& Target, M. (2002). Affect Regulation, Mentalization and the Development of the Self. New York: Other Press. 
Gazzillo, F. (2006). La valutazione della personalità e la ricerca in psicoterapia. In N. Dazzi, V. Lingiardi \& A. Colli (Eds.), La ricerca in psicoterapia. Modelli e strumenti (pp. 609-630). Milano: Raffaello Cortina.

George, C., Kaplan, M., \& Main, M. (1985). Adult Attachment Interview. Unpublished manuscript, University of California, Berkeley.

Grice, H.P. (1975). Logic and Conversation. In P. Cole \& J.L. Morgan (Eds.), Syntax and Semantics volume 3: Speech Acts. New York: Academic Press, 41-58.

Gruppo OPD (1996/2001). La Diagnosi Psicodinamica Operazionalizzata (ed. it. a cura di A. De Coro), Masson, Milano 2002.

Iberni, E. (2010). La coerenza narrativa nell'Adult Attachment Interview: Uno studio per l'adattamento italiano del Coherence Q-Sort. Tesi di dottorato in Psicologia Dinamica, Clinica e dello Sviluppo. "Sapienza" Università di Roma.

Iberni, E., Crisafulli, V., \& De Coro, A. (2007). Diagnosi psicodinamica e valutazione dello stato della mente relativo all'attaccamento: un protocollo per l'assessment e la pianificazione dell'intervento psicoterapeutico nell'ambito del servizio pubblico. Rivista di Psicologia Clinica, 1.

Iberni, E., Vicari, A., Tagini, A., \& Pazzagli, C., (2004, Giugno). Psychodynamic diagnosis and evaluation of current mental state in relation to attachment: a joint application of OPD (Operationalisierte Psychodynamische Diagnostik) and Adult Attachment Interview for the assessment and planning of the psychotherapeutic intervention in the framework of public mental health service. Paper presented at the SPR International Conference, Roma.

Kernberg, O.F. (1984). Disturbi gravi della personalità. Tr. it. Bollati Boringhieri, Torino 1987.

Kernberg, O.F., \& Clarkin, J.F. (1995). The Inventory of Personality Organization. White Plains, NY: The New York Hospital-Cornell Medical Center.

Klagsbrun, M., \& Bowlby, J. (1976). Responses to Separation from Parents: A Clinical Test for Young Children. Projective Psychology, 21, 7-27.

Lenzenweger, M.F., Clarkin, J.F., Kernberg, O.F., \& Foelsch, P., (2001). The Inventory of Personality Organization: Psychometric Properties, Factorial Composition, and Criterion Relations With Affect, Aggressive Dyscontrol, Psychosis Proneness, and Self-Domains in a Nonclinical Sample. Psychological Assessment, 13(4), 577-591.

Lingiardi, V. (2006). La valutazione dei meccanismi di difesa. In N. Dazzi, V. Lingiardi \& A. Colli (Eds.), La ricerca in psicoterapia. Modelli e strumenti (pp. 463-504). Milano: Raffaello Cortina.

Lingiardi, V., Shedler, J., \& Gazzillo, F. (2006). Assessing Personality Change in Psychotherapy with the SWAP-200: A case study. Journal of Personality Assessment, 86, 36-45.

Main, M., \& Goldwyn, R. (1998). Adult Attachment Classification System. Unpublished manuscript. University of California: Berkeley, CA. 
Main, M., Hesse, E., \& Goldwyn, R. (2008). Studying differences in language usage in recounting attachment history, An introduction to the AAI. In H. Steele \& M. Steele (Eds.), Clinical applications of the adult attachment interview (pp. 31-68). New York: Guildford Press.

Mariani, R. (2009). Per uno studio del Processo Referenziale in Psicoterapia Il Dizionario delle Parole Riflessive (IREF) un indicatore linguistico in relazione all' IWRAD nello studio delle narrative. Tesi di Dottorato in Psicologia Dinamica, Clinica e dello Sviluppo. "Sapienza" Università di Roma.

Mariani, R., Gazzillo, F., Lingiardi, V., \& Bucci, W. (2009, Settembre). Verso un'integrazione tra personalità e attività referenziale: uno studio pilota su un caso singolo valutato con SWAP e RA. Relazione presentata al Convegno AIP, Chieti.

Mariani, R., Pazzagli, C., Crisafulli, V., \& De Coro, A. (2010, Settembre). Relazione tra Attaccamento e Misure Linguistiche dell'Attività Referenziale: uno studio sulle narrative in età evolutiva. Relazione presentata al Convegno AIP, sez. Clinica, Torino.

Maskit, B. (2004). Development of the Italian Weighted Referential Activity Dictionary: Linguistic Implications and Clinical Applications. Unpublished paper presented at the 35th Annual Meeting.

Matarrese, A. (2007). La valutazione dell'efficacia delle psicoterapie individuali nel Servizio Pubblico. Uno studio pilota per la messa a punto di un protocollo di ricerca. Tesi di Dottorato in Psicologia Dinamica, Clinica e dello Sviluppo. "Sapienza" Università di Roma.

Muscetta, S., Dazzi, N., De Coro, A., Ortu, F., \& Speranza, A.M. (1999). The attitude toward attachment and change in psychotherapeutic relationship: A study of the coherence of transcript in a short-term psychotherapy with an adolescent. Psychoanalytic Inquiry, 19, 885-921.

Rivolta, L., Mariani, R., \& Tagini, A. (2009, October). The italian reflection (IREF) and affect (IAFF) dictionaries as linguistic indicators of mental functioning. Paper presented at the European Meeting SPR, Bozen, Italy.

Rocco, D., Mariani, R., De Bei, F., Colli, A., \& De Coro, A. (2010, Ottobre). Analisi multidimensionale del processo in una psicoterapia dinamica breve. Relazione presentata al Convegno SPR, Perugia.

Roisman, G.I., Fraley, R.C., \& Belsky, J. (2007). A taxometric study of the Adult Attachment Interview. Developmental Psychology, 43(3), 675-686.

Ruini, C., Ottolini, F., Rafanelli, C., Tossani, E., Ryff, C.D., \& Fava, G.A. (2003). The relationship of psychological well-being to distress and personality. Psychotherapy and Psychosomatics, 72, 268-275.

Safran, J.D., \& Muran, C. (2000). Negotiating the therapeutic alliance: A relational treatment guide. New York: Guilford Press (tr. it. Teoria e pratica dell'alleanza terapeutica, Laterza, Bari 2003).

Speranza, A.M. (2006). La valutazione dell'attaccamento. In N. Dazzi, V. Lingiardi \& A. Colli (Eds.), La ricerca in psicoterapia. Modelli e strumenti (pp. 539-565). Milano: Raffaello Cortina. 
Steiner, H., Araujo, K.B., \& Koopman, C. (2001). The response evaluation measure-71: A new instrument for the measurement of defenses in adult and adolescents. American Journal of Psychiatry, 158, 467-473.

Task Force OPD (2006). OPD-2: Diagnosi Psicodinamica Operazionalizzata (ed. it. a cura di E. Fava et al.), Franco Angeli, Milano 2009.

Westen, D., \& Shedler, J. (2000). Un approccio alla diagnosi della personalità basato sulla comparazione con dei prototipi. In D. Westen, J. Shedler \& V. Lingiardi (Eds.), La valutazione della personalità con la SWAP-200 (pp. 125145). Milano: Raffaello Cortina.

\begin{abstract}
The aim of this work is to show the main results of a series of studies about empirical assessment of outcome and process in Public Services psychotherapies. In the first part are summarized methods and results of a complex longitudinal research project - which included formation of and restitution to the clinical operators - carried out by "Sapienza" University together with the Mental Health Services of a territorial unit of Public Health in Rome. Some research studies focused on therapeutic change and process will follow, through narrative empirical instruments applied to psychotherapies in a Clinical University Centre. The studies here set out are mainly addressed to an improvement of clinical services, to self-monitoring of psychotherapists and to the construction of a theoretic model which may enhance therapeutic effectiveness.
\end{abstract}

\title{
Key words
}

Psychotherapies in public institutions, outcome assessment, process research, narratives, personality disorders 